\title{
MORPHOLOGICAL CHANGES IN THE PROPRIOCEPTORS OF HUMAN ANTERIOR CRUCIATE LIGAMENT DURING DEGENERATIVE OSTEOARTHRITIS
}

\author{
Iskren Gerasimov ${ }^{1,2}$, Stefan Trifonov ${ }^{1}$, Desislava Marinova ${ }^{1}$, Aleksandar Todorov \\ ${ }^{1}$, Iliyana Vacheva ${ }^{1}$, Savelina Popovska ${ }^{3}$, Asparuh Asparuhov ${ }^{2}$, Ivan Ivanov ${ }^{3}$ \\ 1) Department of Anatomy, Cytology, Histology and Biology, Faculty Medicine, \\ Medical University Pleven, Bulgaria. \\ 2) Department of Orthopaedics and Trauma, Faculty Medicine, Medical \\ University Pleven, Bulgaria \\ 3) Department of Pathology, Faculty Medicine, Medical University Pleven, \\ Bulgaria.
}

\section{ABSTRACT}

Introduction/Aim: The anterior cruciate ligament (ACL) of the knee joint is one of the most investigated structures of the musculoskeletal system. Numerous studies are dedicated to the macro- and micro anatomy of human and animal ACL, but not many are focused on the proprioceptors of the ligament. The aim of this study was to establish a relationship between the proprioceptors and the stage of degenerative changes in the ACL.

Materials and Methods: Sixty ACL were harvested from patients admitted for knee replacement surgery. The samples were processed, histochemically and immunohistochemically stained according to standard laboratory protocols. Results were analysed and interpreted. The research was approved by the Ethics Committee of Medical University Pleven.

Results: Fifty-seven patients were included in our study - 37 females and 20 males. The average age of patients was 66.48 years. Degeneration of ACL was classified in four stages, and four different patterns of degeneration were observed: 1) necrosis and inflammation; 2) myxoid and cystic formation; 3) vascularisation with myxoid changes; 4) inflammation and hyalinosis. With the increasing severity of degeneration, the number of specialized nervous structures diminish.

Discussion: Our findings support the results from studies on animal models, conducted by other authors. Cabuk et al. reported a diminishing number of proprioceptors in the PCL of an osteoarthritic human knee. The lower number of proprioceptors could result in the onset or progression of the arthritic process.

Conclusion: Osteoarthritis involves all elements of the knee joint. Altered proprioception could be the reason for unsatisfied patients with artificial knees.

Keywords: ACL, proprioceptors, osteoarthritis,

\section{INTRODUCTION/AIM:}

The anterior cruciate ligament (ACL) of the knee joint is one of the most investigated structures of the musculoskeletal system, that continues to provoke debate between scientists and surgeons. It is thick, stout, intra-articular, but an extra synovial structure which acts to control rotational movement and anterior translation of the femur upon the tibia. ACL originates from a broad anterior intercondylar area of the tibia, passes from anterior to posterior, and inserts into the most posterior part of the medial aspect of the lateral femoral condyle. It is approximately $35 \mathrm{~mm}$ long and $11 \mathrm{~mm}$ in diameter on average. It receives its blood supply from the middle genicular artery and is exclusively innervated by the tibial nerve [1-3].

Numerous studies about macro- and micro anatomy of human or animal ACL can be found in the literature, but not many focused-on proprioceptors exist. Proprioceptors are specialized structures that detect changes in tension, acceleration, the direction of movement and the position of the knee joint. In one of the earliest, detailed light microscopic study in 1967, Freeman and Wyke [4] proposed classificational system of mechanoreceptors, that was modified by Hagert [5] in 2008:

- Type I (Ruffini corpuscle) nerve endings are slow to adapt and have low mechanical thresholds that can signal a static joint's position, the intra-articular pressure and the amplitude and velocity of a movement (Fig. 1).

- Type II (Pacini corpuscle) nerve endings are lowthreshold and fast-adapting nerve endings that are responsible for signalling the acceleration and deceleration of joint rotation (Fig. 2).

- Type III (Golgi-Mazzoni corpuscle) nerve endings are low-threshold and slow-adapting nerve endings that can measure the tension of ligaments when the joint is at the extremes of its movement range (Fig. 3).

- Type IV (free) nerve endings are high-threshold 
pain receptors that become activated when they are subjected to abnormal mechanical deformations, such as knee arthritis or inflammatory processes.

- Type V (unclassified) nerve endings cannot be classified as one of the previously described categories.

The ultrastructure of proprioceptors was examined and documented by Halata [6] in 1976 under an electron microscope.

- Ruffini corpuscles are chiefly near blood vessels, subsinovially. They are 300-800 $\mu \mathrm{m}$ long and approximately $300 \mu \mathrm{m}$ wide. They are composed of 2-6 cylinders, each consisting of an afferent axon with its dendritic processes and terminal processes, Schwann cells, endoneural connective tissue and capsule.

- The second type of nerve ending in the knee joint capsule is represented by encapsulated corpuscles with an inner core (Pacinian corpuscles). They are situated near blood vessels. The corpuscles are either single or in groups of up to five. In rare cases, a corpuscle is actually inside a nerve fibre fascicle. In such cases, the corpuscles do not have a capsule of their own but are enveloped in the perineurium of the nerve. The encapsulated corpuscles with an inner bulb are oblong, being 20-40 $\mu \mathrm{m}$ wide and 150$250 \mu \mathrm{m}$ long. Each corpuscle consists of an afferent axon, a lamellar system of Schwann cells (inner core), a space between the inner bulb and the capsule (subcapsular space) and a capsule which is a continuation of the perineurium of the nerve fascicle.

Osteoarthritis is a process that involves all components of the knee joint - bone, cartilage, soft tissue, including the ACL, blood vessels and nerve endings. Degeneration of the ACL is characterized with loose connective tissue proliferation, cyst formation, myxoid tissue, chondromatosis, crystals of calcium pyrophosphate, necrosis and hyalinosis [7]. Unlike aging, degeneration is characterized by increased cellularity due to perivascular cellular groups and chondrocyte - like cells [8]. Allain et al. [9] applied histological method on longitudinal sections to assess degeneration in collagen fibres of ACL, based on the volume of the damaged collagen. He describes four stages of degeneration in ACL.

The aim of this study is to establish a relationship between the proprioceptors and the stage of degenerative changes of the ACL.

\section{MATERIALS AND METHODS:}

Sixty ACL were harvested from patients admitted for knee replacement surgery in University Hospital "Georgi Stranski" - Pleven and County Hospital "Teodosi Vitanov" - Tryavna between September 2018 and March 2019 after signing informed consent. Excluding criteria, established by us were previous surgical interventions, medical history of rheumatoid arthritis or other autoimmune diseases affecting the collagen, multiple corticosteroid applications (more than one). Samples of more than $1 \mathrm{~cm}^{2}$ were included in our study. The ACL's were removed sharply with a scalpel, cutted once in the longitudinal axis of the collagen fibres, fixed in $10 \%$ buffered formalin, decalcified in EDTA based solution and embedded in paraffin. Serial sections were cut in $4 \mu \mathrm{m}$ thickness on a standard rotatory microtome and mounted on adhesive slides. Histochemical (Haematoxylin and Eosin, Van Gison) and immunohistochemical (S100 - Dako FLEX Poly Rb a, RTU, Cat. No IR50461-2; NFP Dako FLEX Mab a Hu, cl 2F11, RT, Cat, No IR60761-2; Visualisation system - Dako EnVision FLEX, High pH, (Link), Cat No. K800021-2) staining was performed according to standard laboratory protocols in specific order -3 slides for Haematoxylin and Eosin, 1 slide for Van Gison, 1 slide for S100 or NFP, totally $20 \mu$ m thickness. We maintained that sequence in order not to miss the single Pacini corpuscles. Our team adapted combined histochemical (PAS/Alcian - BioGnost) and immunohistochemical (S100 - Dako) staining protocol based on the recommendations of the manufactures to preserve tissue and assess both degeneration changes in the ACL and receptor count. We performed immunohistochemical staining (S100) first according to the standard protocol. After visualisation the samples were: 1) processed with Alcian Blue $\mathrm{pH}$ 2,5 for 45 min.; 2) followed by pouring of the Alcian Blue without washing; 3) incubated in Sodium tetraborate for 10 min.; 4) washed with distillate water; 5) stained with Nuclear Fast Red (Kernechtrot); 6) washed with distillate water; 7) incubated in Periodic Acid (10 min); 8) washed with distillate water and dried; 9) incubated in Shiff reagent (15 min); 10) washed with distillate water; 11) stained with Haematoxylin $(2,5-5 \mathrm{~min}) ; 12)$ washed with water (5 $\mathrm{min}) ; 13)$ dried and covered. Quality of slides was assessed both by internal (fat tissue) and external control (large intestine). Results were analysed and interpreted independently by three specialists in anatomy, cytology and histology and two specialists in pathology. X square test and Anova were used for statistical analyse. The research was approved by the Ethics Committee of Medical University Pleven (No 506/18. 04. 2018).

\section{RESULTS:}

Fifty-seven patients were included in our study - 37 females and 20 males. Three samples were excluded due to technical issues - poor fixation. The average age of patients was 66.48 years. Degeneration of ACL was classified in four stages according to Allain et al. [9] histological method: stage 0 - normal; stage 1 - degenerative lesions in less than $1 / 3$ of the ligament thickness (Fig. 4, 7); stage 2 - degenerative lesions between $1 / 3$ and $2 / 3$ of the ligament thickness (Fig. 5, 8); stage 3 -degenerative lesions in more than 2/3 of the ligament thickness (Fig. 6, 9) (Chart 1). There was no statistically significant difference between the age of the patients when they were divided into different groups, based on the stage of degeneration of the ACL $(\mathrm{F}=0.32, \mathrm{p}=0,8104)$. Four different patterns of degeneration were observed: 1) necrosis and inflammation; 2) myxoid and cystic formation; 3) vascularisation with myxoid changes; 4) inflammation and hyalinosis. The average number of Ruffini corpuscles was $3.57(2-21)$, and the average number of lamellar corpuscles was 0.36 (1-3). With the increasing severity of degeneration, the number of specialized nervous structures diminished - especially in stage 2 and stage 3, where we have found a statistically 
significant reduction of the number of proprioceptors compared to stage 0 and stage $1(\mathrm{X}$ square $=11.46 ; \mathrm{Df}=2$; $\mathrm{p}=0.0032)$ (Table 1).

Fig. 1. Ruffini corpuscle S100/PAS-Alcian blue 400x

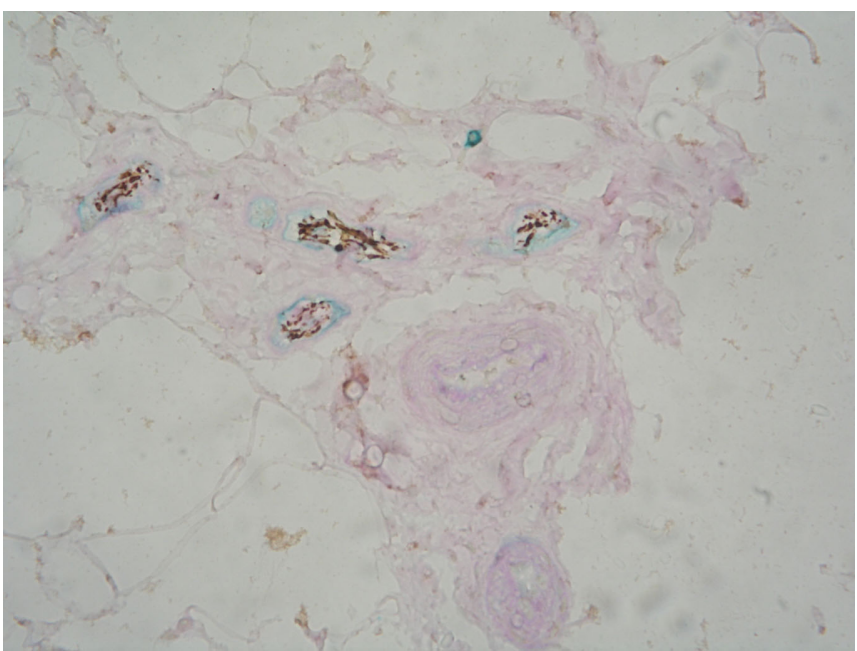

Fig. 2. Pacini corpuscle S100/PAS-Alcian blue 400x

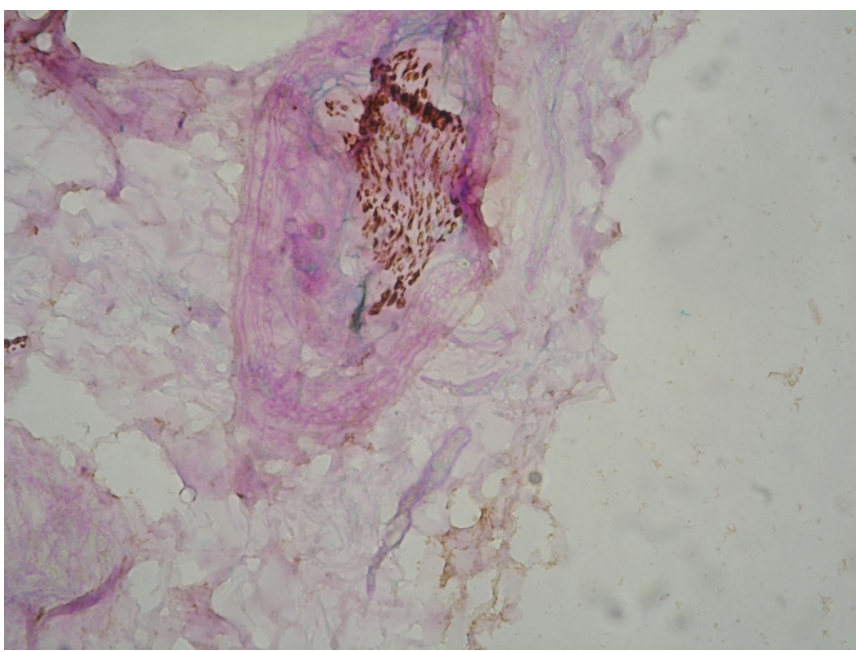

Fig. 3. Golgi - Mazzoni S100/PAS-Alcian blue 400x

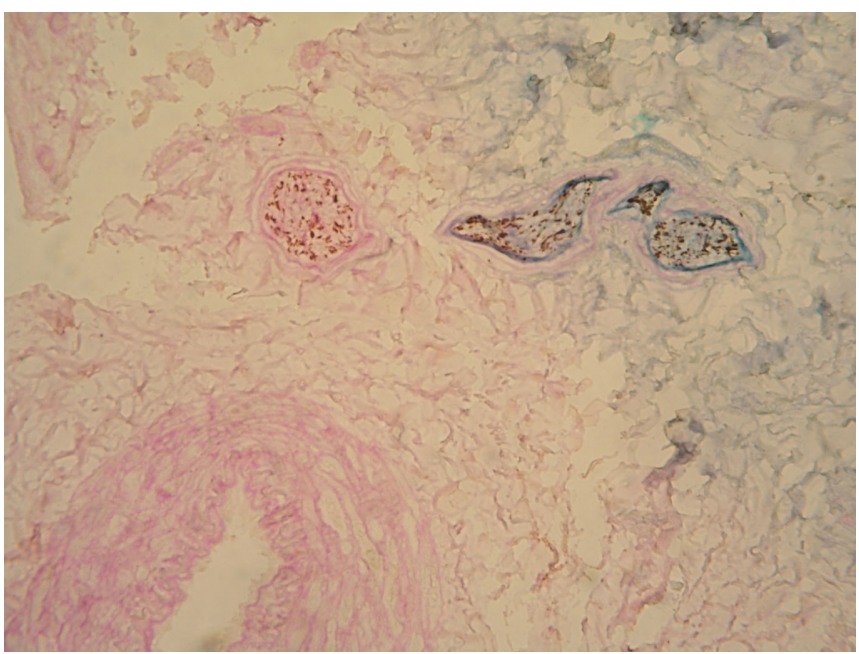

Fig. 4. Stage 1 degenerative lesions in less than $1 / 3$ of the ligament thickness HE 200x

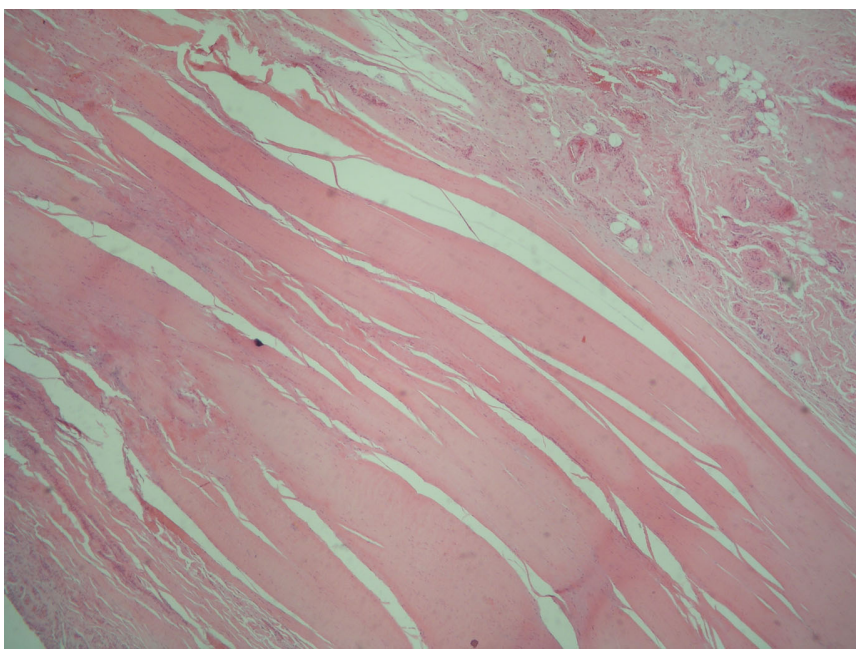

Fig. 5. Stage 2 degenerative lesions between $1 / 3$ and $2 / 3$ of the ligament thickness HE 200x

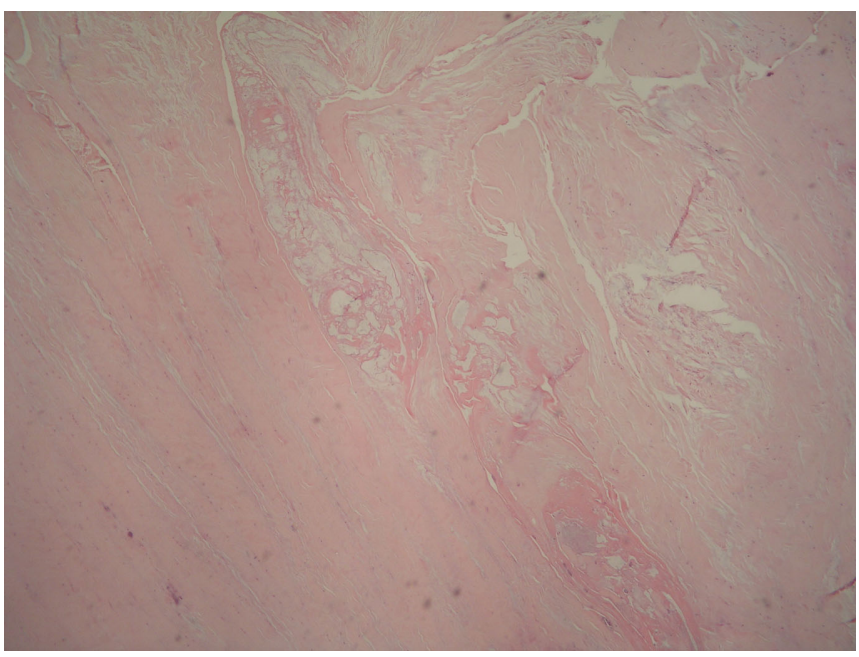

Fig. 6. Stage 3 degenerative lesions in more than $2 /$ 3 of the ligament thickness HE 200x

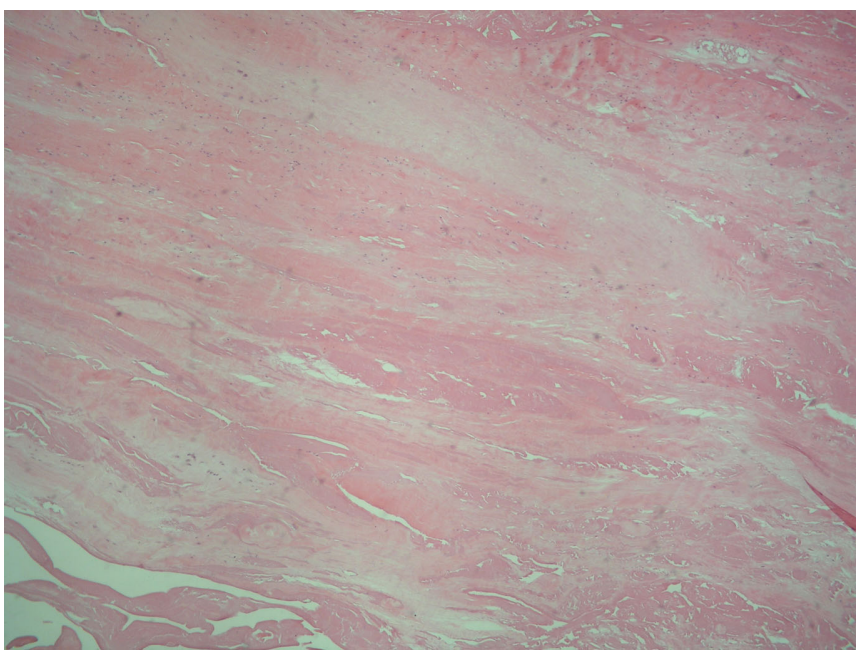


Fig. 7. Stage 1 degenerative lesions in less than $1 / 3$ of the ligament thickness Van Gison 200x

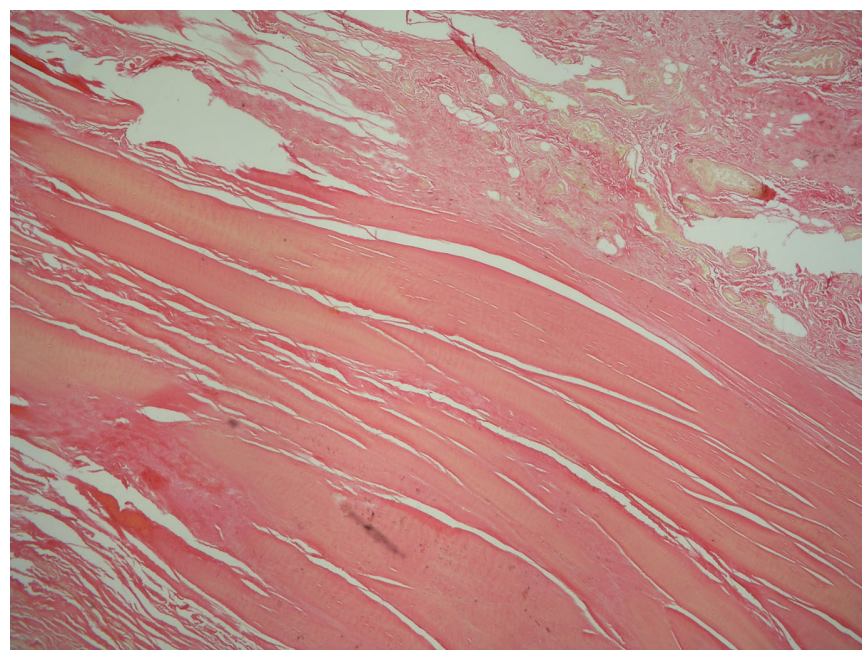

Fig. 8. Stage 2 degenerative lesions between $1 / 3$ and $2 / 3$ of the ligament thickness Van Gison 200x

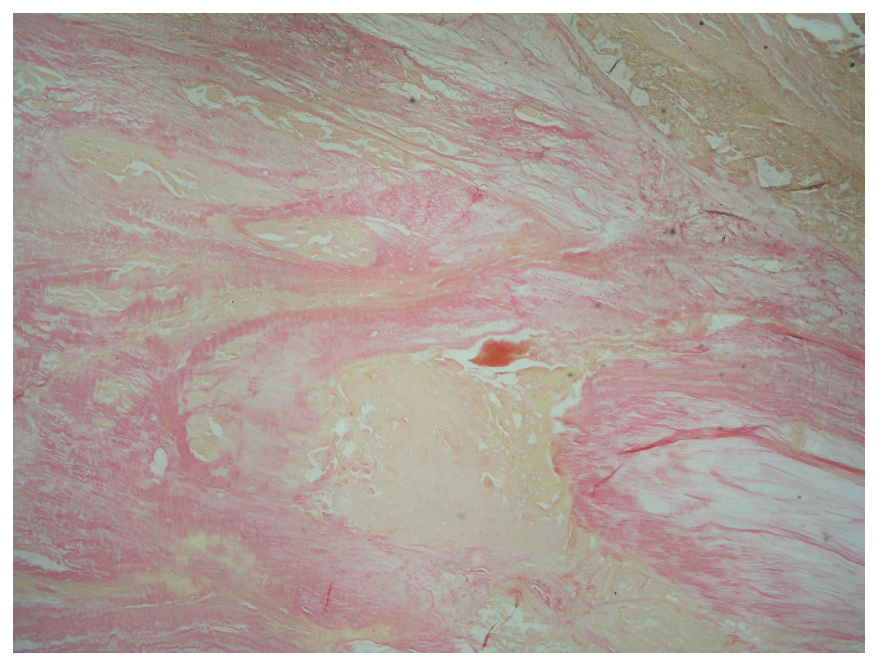

Fig. 9. Stage 3 degenerative lesions in more than $2 /$ 3 of the ligament thickness Van Gison 200x

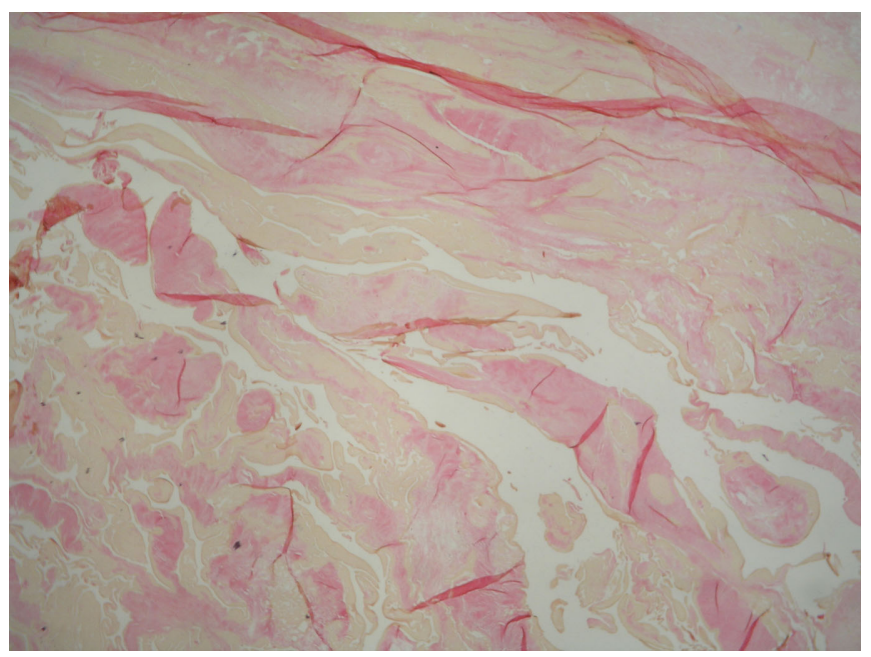

Chart 1. Stage of degeneration in percentage

\section{Stage of degeneration}
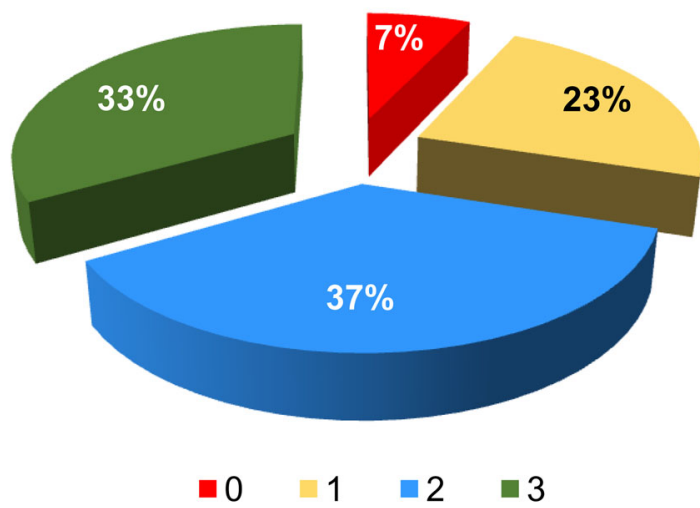

Table 1. Relationship between the stage of degeneration and receptor count

\begin{tabular}{|c|c|c|}
\hline & $\begin{array}{c}\text { Absence of } \\
\text { proprioceptors }\end{array}$ & $\begin{array}{c}\text { Presence of } \\
\text { proprioceptors }\end{array}$ \\
\hline Stage 1 & $3(5.26 \%)$ & $14(24.56 \%)$ \\
\hline Stage 2 & $9(15.79 \%)$ & $12(21.05 \%)$ \\
\hline Stage 3 & $14(24.56 \%)$ & $5(8.77 \%)$ \\
\hline
\end{tabular}

\section{$\mathrm{X}$ - square $=11.46 ; \mathrm{Df}=2 ; \mathrm{p}=0.0032$}

Stage 2 and Stage 3 of degeneration in the ACL are characterized with a statistically significant diminishing of proprioceptors compared to stage 0 and stage 1 .

\section{DISCUSSION:}

Proprioception diminishes with the severity of degeneration, independent from the age and sex in patients affected by osteoarthritis. Proprioceptors are not found only in the ACL, but also in the posterior cruciate ligament, collateral ligaments, joint capsule, etc. One of the reasons for this event could be a decreased number of proprioceptors, that we observed in the current study. Other reasons for altered proprioception could be atrophy of muscles or changes in the central nervous system [10]. Proprioceptors in intact ACL contribute towards functional stability of the knee joint. Degenerative changes in the ACL not only affect the mechanical stability but also lead to loss of neuromuscular control [11].

Histological and immunohistochemical methods proved to be reliable in assessing degeneration of ACL and receptor morphology, as noted by other authors [12-14]. Both immunohistochemical methods (S100 and NFP) provided similar results in our study. We identified different types of nerve endings in the ACL, as described by Halata et al. [6] and Freeman and Wyke [4] - Ruffini corpuscles (main type), Pacini corpuscles (rarely found), GolgiMazzoni corpuscle (rarely found) and free nerve endings 
(2nd most common) in different proportion. We have used the histological method implemented by Allain et al. [9] to access the stage of degeneration and found that single ACL, in different parts can have a different stage of degeneration. Therefor we recommend careful observation of the whole ACL. In our study, the highest present stage of degeneration was recorded. With the increasing stage of degeneration and severity of osteoarthritis, the number of specialized nervous structures diminished. Our findings support the results of Aydog et al. [15] who described a diminishing number of receptors in experimental animal study, but we could not find age related connection in ours. We believe that the severity of degeneration is the main reason for low number of proprioceptors. Çabuk et al. [16] reported diminishing number of proprioceptors in the PCL of osteoarthritic human knee, that is accordance with our findings for ACL.

One weak spot in our study is that we were unable to study proprioceptors in intact healthy ACL. Therefor our results cannot be applied directly to normal ACL.

We found several studies in the literature confirming the presence of proprioceptors in residual strums of thorn ACL's $[11,13,17]$. If ACL remnants play a substantial role in proprioception, healthy or even degenerated ACL with a lower count of proprioceptors may be even more important. Lower number of proprioceptors could result in the onset or progression of arthritic process.

\section{CONCLUSION:}

Osteoarthritis affects the whole knee joint, not only bone but also soft tissues, blood vessels and nerve endings. Severity of the degenerative process leads to a decreasing number of proprioceptors. Altered proprioception could be the reason for unsatisfied patients with artificial knees.

\section{Acknowledgement:}

The research work was funded by Medical University Pleven.

\section{REFERENCES:}

1. Deehan DJ, Cawston TE. The biology of integration of the anterior cruciate ligament. J Bone Joint Surg Br. $2005 \mathrm{Jul} ; 87(7): 889-95$. [PubMed] [Crossref]

2. Bicer EK, Lustig S, Servien E, Selmi TA, Neyret P. Current knowledge in the anatomy of the human anterior cruciate ligament. Knee Surg Sports Traumatol Arthrosc. 2010 Aug; 18(8):1075-84. [PubMed] [Crossref]

3. Tantisricharoenkul G, LindenRosen M, Araujo P, Zhou J, Smolinski $\mathrm{P}, \mathrm{Fu}$ F. Anterior cruciate ligament: an anatomical exploration in humans and in a selection of animal species. Knee Surg Sports Traumatol Arthrosc. 2014 May;22(5):961-71. [PubMed] [Crossref]

4. Freeman R, Wyke B. The innervation of the knee joint. An anatomical and histological study in the cat. J. Anat. 1967 Jun;101(Pt 3):505-32. [PubMed]

5. Hagert E. Wrist ligaments-innervation patterns and ligamentomuscular reflexes [Thesis], [Stockholm]: Karolinska Institutet; 2008; $51 \mathrm{p}$.

6. Halata Z. The ultrastructure of the sensory nerve endings in the articular capsule of the knee joint of the domestic cat (Ruffini corpuscles and
Pacinian corpuscles). J Anat. 1977 Dec;124(Pt 3):717-29. [PubMed]

7. Cushner FD, La Rosa DF, Vigorita VJ, Scuderi GR, Scott WN, Insall JN. A quantitative histologic comparison: ACL degeneration in the osteoarthritic knee. J Arthroplasty 2003; 18(6):687-92. [Crossref]

8. Hasegawa A, Nakahara H, Kinoshita M, Asahara H, Koziol J, Lotz MK. Cellular and extracellular matrix changes in anterior cruciate ligaments during human knee aging and osteoarthritis. Arthritis Res Ther. 2013 Feb 14;15(1):R29. [PubMed] [Crossref]

9. Allain J, Goutallier D, Voisin MC. Macroscopic and histological assessments of the cruciate ligaments in arthrosis of the knee. Acta Orthop Scand. 2001 Jun;72(3):266-9. [PubMed] [Crossref]

10. Han J, Waddington G, Adams R, Anson J, Liu Y. Assessing proprioception: A critical review of methods. J Sport Health Sci. 2016 Mar;5(1):8090. [ubMed] [Crossref]

11. Dhillon MS, Bali K, Vasistha RK. Immunohistological evaluation of proprioceptive potential of the residual stump of injured anterior cruciate ligaments (ACL). Int Orthop. 2010 Jun;34(5):737-41. [PubMed]

\section{[Crossref}

12. Bali K, Dhillon MS, Vasistha RK, Kakkar N, Chana R, Prabhakar S. Efficacy of immunohistological methods in detecting functionally viable mechanoreceptors in the remnant stumps of injured anterior cruciate ligaments and its clinical importance.

Knee Surg Sports Traumatol Arthrosc. 2012 Jan;20(1):75-80. [PubMed] [Crossref]

13. Lee BI, Min KD, Choi HS, Kwon SW, Chun DI, Yun ES, et al. Immunohistochemical study of mechanoreceptors in the tibial remnant of the ruptured anterior cruciate ligament in human knees. Knee Surg Sports Traumatol Arthrosc. 2009 Sep; 17(9):1095-101 [PubMed] [Crossref]

14. Sonnery-Cottet B, Bazille C, Hulet C, Colombet P, Cucurulo T, Panniset JC, et al. Histological features of the ACL remnant in partial tears. Knee. 2014 Dec;21(6):1009-13. [PubMed] [CrossRef]

15. Aydoð ST, Korkusuz P, Doral MN, Tetik O, Demirel HA. Decrease in the numbers of mechanoreceptors in rabbit ACL: the effects of aging. Knee Surg Sports Traumatol Arthrosc. 2006 Apr;14(4):325-9. [PubMed] [Crossref]

16. Çabuk H, Çabuk F, Tekin AC, Dedeoglu SS, Cakar M, Buyukkurt 
CD. Lower numbers of mechanoreceptors in the posterior cruciate ligament and anterior capsule of the osteoarthritic knees. Knee Surg Sports Traumatol Arthrosc. 2017 Oct; 25
(10):3146-3154. [PubMed] [CrossRef]

17. Chun KC, Lee SH, Kim JW, Jin EJ, Kim KM, Chun CH. Immunohistochemical and immunocytochemical study of mechanoreceptors in anterior cruciate ligament reconstruction with the remnant-preserving technique using Achilles tendon allografts. $J$ Orthop Surg Res. 2017 Jun 14;12(1): 93. [PubMed] [Crossref]

Please cite this article as: Gerasimov I, Trifonov S, Marinova D, Todorov A, Vacheva I, Popovska S, Asparuhov A, Ivanov I. Morphological changes in the proprioceptors of human anterior cruciate ligament during degenerative osteoarthritis. $J$ of IMAB. 2019 Oct-Dec;25(4):2847-2852. DOI: https://doi.org/10.5272/jimab.2019254.2847

Received: 22/03/2019; Published online: 19/12/2019

\section{Address for correspondence: \\ Iskren Gerasimov}

Department Anatomy, cytology, histology and biology, Faculty Medicine, Medical University Pleven,

5, Hristo Botev str., Ent, K, ap. 14, Pleven, Bulgaria.

E-mail: is.gerasimov@abv.bg 